\title{
Comparison of HOMA-IR, HOMA- $\beta \%$ and disposition index between US white men and Japanese men in Japan in the ERA JUMP study: was the calculation of disposition index legitimate?
}

\author{
Keishi Yamauchi $^{1} \cdot$ Yuka Sato $^{1} \cdot$ Yasuto Nakasone $^{1} \cdot$ Toru Aizawa $^{1}$
}

Received: 22 March 2015 / Accepted: 7 April 2015 / Published online: 14 May 2015

(C) Springer-Verlag Berlin Heidelberg 2015

Keywords Disposition index $\cdot$ HOMA- $\beta \% \cdot$ HOMA-IR

$\begin{array}{ll}\text { Abbreviations } \\ \text { DI } & \text { Disposition index } \\ \text { FIRI } & \text { Fasting immunoreactive insulin } \\ \text { FPG } & \text { Fasting plasma glucose } \\ \text { HOMA- } \beta \% & \text { HOMA of beta cell function }\end{array}$

To the Editor: Ahuja et al [1] determined insulin resistance and insulin secretion using HOMA-IR and HOMA of beta cell function (HOMA- $\beta \%$ ), respectively, in white men living in the USA and Japanese men in Japan. They elegantly demonstrated that both HOMA-IR and HOMA- $\beta \%$ were significantly higher in the white men compared with the Japanese. In addition, they calculated disposition index (DI, in this case, oral disposition index) as a measure of beta cell function, i.e. HOMA- $\beta \%$ with the degree of insulin resistance being taken into consideration [2]. On the basis that the DI was higher in white than in Japanese people, the authors concluded that the higher DI in white men 'may partly explain lower susceptibility of white people than Japanese people to developing type 2 diabetes'. We are afraid that there was a serious problem in the calculation of DI, and

Toru Aizawa

taizawax@ai-hosp.or.jp

1 Diabetes Center, Aizawa Hospital, 2-5-1, Honjo, Matsumoto 390-8510, Japan therefore the conclusion above may not be scientifically sound. The reason for this is as follows. In this study, DI was calculated as HOMA- $\beta \%$ divided by HOMA-IR, which is problematic. $\mathrm{HOMA}-\beta \%=(\mathrm{FIRI} \times 20) /(\mathrm{FPG}-3.5)[3]$, where FIRI denotes fasting immunoreactive insulin $(\mu \mathrm{U} / \mathrm{ml})$ and FPG denotes fasting plasma glucose $(\mathrm{mmol} / \mathrm{l})$. On the other hand, HOMA-IR $=($ FIRI $\times$ FPG $) / 22.5$ [3]. Therefore, the DI defined by the authors, HOMA- $\beta \% /$ HOMA$\mathrm{IR}=[(\mathrm{FIRI} \times 20) /(\mathrm{FPG}-3.5)] /[(\mathrm{FIRI} \times \mathrm{FPG}) / 22.5]$, yields $(20 \times$ $22.5) /\left[(\mathrm{FPG})^{2}-(\mathrm{FPG} \times 3.5)\right]=450 /\left[(\mathrm{FPG})^{2}-(\mathrm{FPG} \times 3.5)\right]$ (Fig. 1). As can be seen, the DI thus calculated is simply a function of FPG, not an index of beta cell function. Therefore the DI calculated in the paper of Ahuja et al [1] was naturally higher in whites than in the Japanese individuals because FPG was lower in the former than in the latter. It is obvious that one cannot a priori assume that individuals with a low FPG have robust beta cell function and vice versa. The idea of defining DI as HOMA- $\beta \% / \mathrm{HOMA}$ IR might have arisen from the authors' understanding that 'DI is assumed to be hyperbolic (the constant product of insulin sensitivity and insulin secretion) for individuals with the same degree of glucose tolerance,' which is not always correct $[4,5]$. A hyperbolic relationship between indices of insulin sensitivity (SI) and insulin secretion exists for a certain combination of the two, but not for all combinations $[4,5]$. There is certainly ethnic diversity, as inferred by Simonson et al [6], such that the relationship between the two in the Japanese population is different from that in white people [5]. It is strongly recommended that the legitimacy of the calculation of DI using any combination of indices of SI (or IR) and insulin secretion should be verified, before utilising the DI value as a measure of beta cell function. 


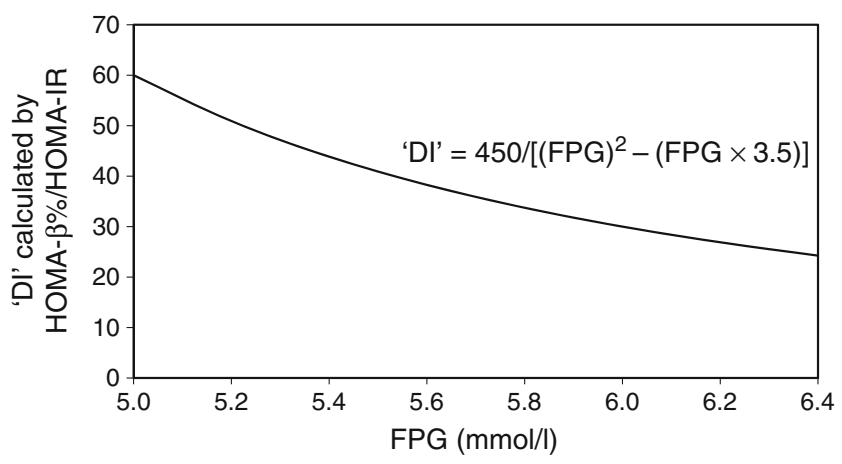

Fig. 1 DI calculated by HOMA- $\beta \% /$ HOMA-IR is simply a function of fasting plasma glucose

Funding None.

Duality of interest The authors declare that there is no duality of interest associated with this manuscript.

Contribution statement KY, YS, YN and TA contributed to conception and design. TA drafted the article. All authors critically revised the article for intellectual content and approved the final version. KY and TA are guarantors of this work.

\section{References}

1. Ahuja Y, Kadowaki T, Evans RW et al (2015) Comparison of HOMA-IR, HOMA- $\beta \%$ and disposition index between US white men and Japanese men in Japan: the ERA JUMP study. Diabetologia 58:265-271

2. Utzschneider KM, Prigeon RL, Faulenbach MV et al (2009) Oral disposition index predicts the development of future diabetes above and beyond fasting and 2-h glucose levels. Diabetes Care 32:335341

3. Matthews DR, Hosker JP, Rudenski AS, Naylor BA, Treacher DF, Turner RC (1985) Homeostasis model assessment: insulin resistance and $\beta$-cell function from fasting plasma glucose and insulin concentrations in man. Diabetologia 28:412-419

4. Retnakaran R, Shen S, Hanley AJ, Vuksan V, Hamilton JK, Zinman B (2008) Hyperbolic relationship between insulin secretion and sensitivity on oral glucose tolerance test. Obesity 16:1901-1907

5. Sato Y, Oka R, Nakasone Y, Katakura M, Yamauchi K, Aizawa T (2015) Impact of one-hour postchallenge glucose on the relationship between insulin sensitivity and secretion. Endocr $\mathrm{J}$ (in press)

6. Simonson DC (2015) Surrogate measures of insulin resistance: does one size fit all? Diabetologia 58:207-10 